\title{
Five-quark components in baryons
}

\author{
Bing-Song Zou \\ Institute of High Energy Physics, CAS, P.O.Box 918, Beijing 100049, China ; \\ Theoretical Physics Center for Science Facilities, CAS, Beijing 100049, China; \\ Center of Theoretical Nuclear Physics, National Lab of Heavy Ion Accelerator, Lanzhou 730000, China
}

\begin{abstract}
Evidence has been accumulating for the existence of significant intrinsic non-perturbative fivequark components in various baryons. The inclusion of the five-quark components gives a natural explanation of the excess of $\bar{d}$ over $\bar{u}$, significant quark orbital angular momentum in the proton, the problematic mass and decay pattern of the lowest $1 / 2^{-}$baryon nonet, etc.. A breathing mode of $q q q \leftrightarrow q q q q \bar{q}$ is suggested for the lowest $1 / 2^{-}$baryon octet. Evidence of a predicted member of the new scheme, $\Sigma^{*}\left(1 / 2^{-}\right)$around $1380 \mathrm{MeV}$, is introduced.
\end{abstract}

Key words: Baryon, five-quark components

PACS: 14.20.-c, 12.39.-x

\section{5-quark components in the proton}

In the classical quark models prior to QCD, each proton was regarded as composed of two $u$ quarks and one $d$ quark. The classical quark models gave very good description of the mass pattern and magnetic moments for the baryon $\mathrm{SU}(3)$ baryon $1 / 2^{+}$octet and $3 / 2^{+}$decuplet of spatial ground states. Then the QCD introduces the gluon field which can fluctuate into sea quark $q \bar{q}$ pairs in addition to the three valence quarks inside a proton. But until about the year of $1992, \bar{u}(x)=\bar{d}(x)$ and $s(x)=\bar{s}(x)$ were assumed for the sea quark parton distribution functions.

However, evidence has been accumulating for the existence of significant intrinsic nonperturbative five-quark components in the proton. A surprisingly large asymmetry between the $\bar{u}$ and $\bar{d}$ sea quark distributions in the proton with $\bar{d}-\bar{u} \sim 0.12$ was observed from deep inelastic scattering and Drell-Yan experiments [1]. A popular explanation for the excess of $\bar{d}$ over $\bar{u}$ in the proton was given by meson-cloud model [2] by including in the proton a mixture of $n \pi^{+}$with the $\pi^{+}$composed of $u \bar{d}$. Recently, by studying strangeness in the proton, a new configuration of diquark-diquark-antiquark structure is proposed for the five-quark components in the proton [3]. The diquark cluster configurations also give a natural explanation for the excess of $\bar{d}$ over $\bar{u}$ in the proton with a mixture of $[u d][u d] \bar{d}$ component in the proton [3]. The measured value of $\bar{d}-\bar{u} \sim 0.12$ demands the 5-quark components in the proton to be more than $12 \%$.

Further evidences came from proton spin problem and single-spin asymmetry problem, which both indicate the presence of quark orbital angular momentum in the proton. The five-quark $u u d q \bar{q}$ components in the proton with the $\bar{q}$ of negative intrinsic parity demand either a quark or anti-quark in the P-wave to make up the proton of positive intrinsic parity. So it gives naturally the nonzero quark orbital angular momentum and hence a natural explanation to both problems [3, 4]. 
To tell the relative importance of meson cloud and diquark cluster mixtures in the proton, more precise experiments on the strangeness in the proton are needed. While the $K \Lambda$ meson cloud picture predicts strangeness magnetic moment and strangeness radius both negative [5], the diquark cluster picture predicts both positive [3]. Present experiments are not precise enough to distinguish the two pictures [6, 7]. The extremely smallness of these strangeness observables may either indicate very small percentage of the strangeness component in the proton or about equally the $K \Lambda$ meson cloud and $[u d][u s] \bar{s}$ contributions canceling each other.

\section{New scheme for $N^{*}(1535)$ and its $1 / 2^{-}$nonet partners with large 5-quark components}

In the simple $3 \mathrm{q}$ constituent quark model, the lowest spatial excited baryon is expected to be a $(u u d) N^{*}$ state with one quark in orbital angular momentum $L=1$ state, and hence should have negative parity. Experimentally, the lowest negative parity $N^{*}$ resonance is found to be $N^{*}(1535)$, which is heavier than two other spatial excited baryons: $\Lambda^{*}(1405)$ and $N^{*}(1440)$. This is the long-standing mass reverse problem for the lowest spatial excited baryons.

Recently a large value of $g_{N^{*}(1535) K \Lambda}$ is deduced [8, 9] by a simultaneous fit to BES data on $J / \psi \rightarrow \bar{p} p \eta, p K^{-} \bar{\Lambda}+c . c$, and COSY data on $p p \rightarrow p K^{+} \Lambda$. There is also evidence for large $g_{N^{*}(1535) N \eta^{\prime}}$ coupling from $\gamma p \rightarrow p \eta^{\prime}$ reaction at CLAS [10] and $p p \rightarrow p p \eta^{\prime}$ reaction [11], and large $g_{N^{*}(1535) N \phi}$ coupling from $\pi^{-} p \rightarrow n \phi, p p \rightarrow p p \phi$ and $p n \rightarrow d \phi$ reactions [12, 13], but smaller coupling of $g_{N^{*}(1535) K \Sigma}$ from comparison of $p p \rightarrow p K^{+} \Lambda$ to $p p \rightarrow p K^{+} \Sigma^{0}$ [14].

The mass reverse problem can be easily understood by considering 5 -quark components in them [8, 15]. The $N^{*}(1535)$ could be the lowest $L=1$ orbital excited $\mid u u d>$ state with a large admixture of $\mid[u d][u s] \bar{s}>$ pentaquark component having $[u d],[u s]$ and $\bar{s}$ in the ground state. The $N^{*}(1440)$ could be the lowest radial excited $\mid u u d>$ state with a large admixture of $\mid[u d][u d] \bar{d}>$ pentaquark component having two $[u d]$ diquarks in the relative P-wave. While the lowest $L=1$ orbital excited $\mid u u d>$ state should have a mass lower than the lowest radial excited $\mid u u d>$ state, the $\mid[u d][u s] \bar{s}>$ pentaquark component has a higher mass than $\mid[u d][u d] \bar{d}>$ pentaquark component. The lighter $\Lambda^{*}(1405) 1 / 2^{-}$is also understandable in this picture. Its main 5-quark configuration is $\mid[u d][u s] \bar{u}>$ which is lighter than the corresponding 5-quark configuration $\mid[u d][u s] \bar{s}>$ in the $N^{*}(1535) 1 / 2^{-}$.

The large mixture of the $\mid[u d][u s] \bar{s}>$ pentaquark component in the $N^{*}(1535)$ may also explain naturally its large couplings to the $N \eta, N \eta^{\prime}, N \phi$ and $K \Lambda$ together with its small couplings to the $N \pi$ and $K \Sigma$. In the decay of the $\mid[u d][u s] \bar{s}>$ pentaquark component, the $[u d]$ diquark with isospin $I=0$ is stable and keeps unchanged while the $[u s]$ diquark is broken to combine with the $\bar{s}$ to form either $K^{+}(u \bar{s}) \Lambda([u d] s)$ or $\phi(s \bar{s}) p([u d] u)$.

The inclusion of the large 5-quark components in the $N^{*}(1535)$ causes a natural cancelation between the contributions of the $q q q$ and $q q q q \bar{q}$ components to the axial charge of the $\mathrm{N}(1535)$ resonance [16] and introduces an important new mechanism as shown in Fig. 11 for its electromagnetic transition $\gamma^{*} N \rightarrow N^{*}(1535)$ [17].

The experimental measured $\gamma^{*} N \rightarrow N^{*}(1535)$ amplitude has a much flatter $Q^{2}$-dependence than the classical $q q q$ quark model predictions as shown in Fig. 2 (left). The new mechanism gives a much flatter $Q^{2}$-dependence as shown by the dot-dashed curve in Fig. 2 With about $45 \%$ 5-quark components in $N^{*}(1535)$ and $20 \%$ in proton, the measured electromagnetic transition $\gamma^{*} N \rightarrow N^{*}(1535)$ amplitude is much better fitted as shown in Fig.2(right).

Taking the $q q q q \bar{q}$ components into account, the constituent quarks masses should be a bit smaller than the ones employed in the case of pure $q q q$ configuration. To reproduce the mass 


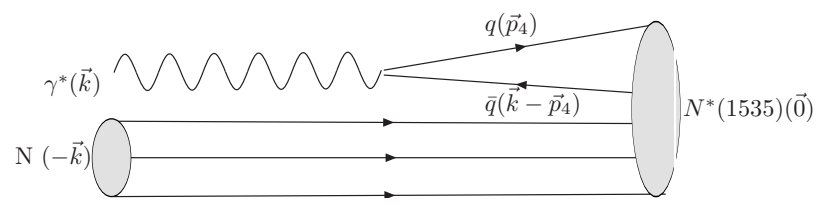

Figure 1: $\gamma^{*} \rightarrow q \bar{q}$ mechanism for the $\gamma^{*} N \rightarrow N^{*}(1535)$ transition.
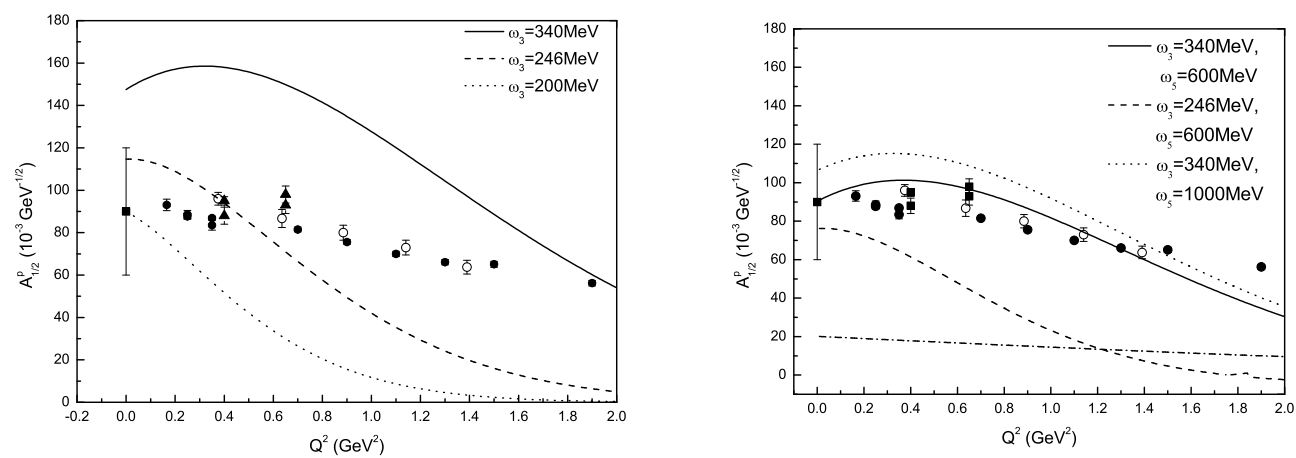

Figure 2: The helicity amplitude $A_{1 / 2}^{p}$ for $\gamma^{*} p \rightarrow N^{*}$ (1535) with pure $q q q$ configuration (left) and with 5-quark component in addition (right) where the dot-dashed curve is the contribution from the $\gamma^{*} \rightarrow q \bar{q}$ transition [17].

for the nucleon when the five-quark components have been included, the values $m_{u}=m_{d}=$ $m=290 \mathrm{MeV}$ and $m_{s}=430 \mathrm{MeV}$ are used. The oscillator parameter for the $q q q$ components, $\omega_{3}$, is from literature, basically determined from the proton radius. The oscillator parameter for the $q q q q \bar{q}$ components, $\omega_{5}$, is treated as a free parameter, which is found to be larger than $\omega_{3}$. This is consistent with other empirical evidence favoring larger value of the $\omega_{5}[18,19$, 20, 21]. In our extended quark model with each baryon as a mixture of the three-quark and five-quark components, the two components represent two different states of the baryon. For the $q q q q \bar{q}$ state, there are more color sources than the $q q q$ state, and may make the effective phenomenological confinement potential stronger. For the lowest $1 / 2^{-}$baryon octet, the larger $\omega_{5}$ value corresponds a smaller size for the 5-quark components. An intuitive picture for our extended quark model is the $q q q \leftrightarrow q q q q \bar{q}$ breathing mode like this: the $q q q$ state with $L=1$ and higher kinetic energy has weaker potential; when quarks expand, a $q \bar{q}$ pair is pulled out and results in a $q q q q \bar{q}$ state with $L=0$ and stronger potential; the stronger potential leads $q q q q \bar{q}$ state shrinking to a more compact state which then makes the $\bar{q}$ to annihilate with a quark easily and transits to the $q q q$ state with $L=1$ and more kinetic energy to expand; this leads to constantly transitions between these two states.

If this picture of large 5-quark mixture is correct, there should also exist the SU(3) nonet partners of the $N^{*}(1535)$ and $\Lambda^{*}(1405)$, i.e., an additional $\Lambda^{*} 1 / 2^{-}$around $1570 \mathrm{MeV}$, a triplet $\Sigma^{*} 1 / 2^{-}$around $1360 \mathrm{MeV}$ and a doublet $\Xi^{*} 1 / 2^{-}$around $1520 \mathrm{MeV}$ [15]. There is no hint for these baryon resonances in the PDG tables [22]. However, as pointed out in Ref. [23], there is in fact evidence for all of them in the data of $J / \psi$ decays and it can be easily checked with the high 
statistics BESIII data in near future [24]. Recently, new evidence for the predicted $\Sigma^{*}$ resonance with $J^{P}=1 / 2^{-}$and mass around $1380 \mathrm{MeV}$ was found in the old data of $K^{-} p \rightarrow \Lambda \pi^{+} \pi^{-}$ reaction [25], as introduced in the next section.

\section{Evidence for the predicted $\Sigma^{*}\left(1 / 2^{-}\right)$of the new scheme}

The unquenched models give interesting predictions for the isovector partner of the $\Lambda^{*}(1405)$ and $N^{*}(1535)$. While the penta-quark models [15, 26] predict a $\Sigma^{*}\left(1 / 2^{-}\right)$resonance with a mass around or less than its corresponding $\Lambda^{*}$ partner, the meson cloud model [27] predicts it to be nonresonant broad structure. The predictions of these unquenched models and the classical quenched quark models are distinctive and need to be checked by experiments. Hence we re-examined the old data of $K^{-} p \rightarrow \Lambda \pi^{+} \pi^{-}$reaction to see whether there is evidence for its existence or not [25].

The $K^{-} p \rightarrow \Lambda \pi^{+} \pi^{-}$reaction was studied extensively around 30 years ago for extracting properties of the $\Sigma^{*}(1385)$ resonance, with $K^{-}$beam momentum ranging from $0.95 \mathrm{GeV}$ to 8.25 $\mathrm{GeV}$. In the invariant mass spectrum of $\Lambda \pi$ of this reaction there is a strong peak with mass around $1385 \mathrm{MeV}$ and width around $40 \mathrm{MeV}$. The mass fits in the pattern of SU(3) baryon decuplet of $J^{P}=3 / 2^{+}$predicted by the classical quark model perfectly. The angular distribution analyses also conclude that the spin of this resonance is $3 / 2$. However we found that all these analyses are in fact assuming that there is only one resonance under the peak. Nobody has considered that there are probably two resonances there. This may be because there are no other predicted $\Sigma^{*}$ resonances around this mass region in the classical quark models. Since now a new $\Sigma^{*}$ with the $J^{P}=1 / 2^{-}$around this mass region is predicted by various unquenched models, the old $K^{-} p \rightarrow \Lambda \pi^{+} \pi^{-}$reaction should be re-scrutinized carefully.

We examined previous experimental analyses on the $K^{-} p \rightarrow \Lambda \pi^{+} \pi^{-}$reaction. Among them, we find that the invariant mass spectra of $\Lambda \pi^{-}$with beam momentum $P_{K^{-}}=1.0 \sim 1.8 \mathrm{GeV}$ are different from others. The peak around $\Sigma^{*}(1385)$ in these mass spectra cannot be fit as perfect as other sets of data with a single Breit-Wigner resonance. Since Ref.[28] presents the largest data sample and the most transparent angular distribution analysis of this reaction, we re-fit the $\Lambda \pi^{-}$ mass spectrum and angular distribution of Ref.[28] by taking into account the possibility of two $\Sigma^{*}$ resonances in this mass region.

The results of the fits with a single and two $\Sigma^{*}$ resonances around $1385 \mathrm{MeV}$ are shown in Fig. 3 and Table 1 where fitted parameters with statistical errors are given. The fit with a single $\Sigma^{*}$ resonance (Fit1) is already not bad. The fit with two $\Sigma^{*}$ resonances (Fit2) improves $\chi^{2}$ by 10.5 compared with the Fit 1 for 60 data points with 3 more fitting parameters. Although this is just a less than $3 \sigma$ improvement, a point favoring Fit 2 is that while the single $\Sigma^{*}$ resonance in Fit1 has a width larger than the PDG value [22] of $36 \pm 5 \mathrm{MeV}$ for the $\Sigma^{*}(1385)$ resonance, the narrower $\Sigma^{*}$ resonance in Fit2 gives a width compatible with the PDG value for the $\Sigma^{*}(1385)$ resonance. In the Fit2, there is an additional broader $\Sigma^{*}$ resonance with a width about $120 \mathrm{MeV}$.

\begin{tabular}{ccccccc}
\hline \hline & $M_{\Sigma^{*}(3 / 2)}$ & $\Gamma_{\Sigma^{*}(3 / 2)}$ & $M_{\Sigma^{*}(1 / 2)}$ & $\Gamma_{\Sigma^{*}(1 / 2)}$ & $\chi^{2} / n d f$ (Fig.1) & $\chi^{2} / n d f$ (Fig.2) \\
\hline Fit1 & $1385.3 \pm 0.7$ & $46.9 \pm 2.5$ & & & $68.5 / 54$ & $10.1 / 9$ \\
Fit2 & $1386.1_{-0.9}^{+1.1}$ & $34.9_{-4.9}^{+5.1}$ & $1381.3_{-8.3}^{+4.9}$ & $118.6_{-35.1}^{+55.2}$ & $58.0 / 51$ & $3.2 / 9$ \\
\hline \hline
\end{tabular}

Table 1: Fitted parameters with statistical errors and $\chi^{2}$ over number of degree of freedom (ndf) for the fits with a single (Fit1) and two $\Sigma^{*}$ resonances (Fit2) around $1385 \mathrm{MeV}$. 

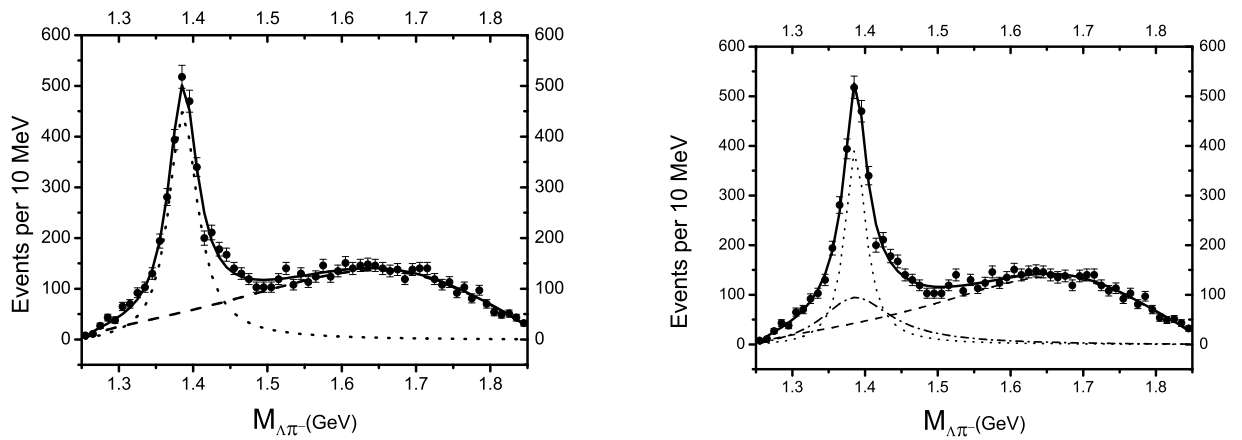

Figure 3: Fits to the $\Lambda \pi^{-}$mass spectrum with a single $\Sigma^{*}$ (left) and two $\Sigma^{*}$ resonances (right) around $1385 \mathrm{MeV}$ with fitting parameters listed in Table 1. The experiment data are from Ref.[28] on $K^{-} p \rightarrow \Lambda \pi^{+} \pi^{-}$with beam momenta around $1.4 \mathrm{GeV}$.

The preferred assignment of $\operatorname{spin} J=3 / 2$ for the $\Sigma^{*}(1385)$ resonance in Ref. [28] is demonstrated by the distribution of the cosine of the angle between the $\Lambda$ direction and the $K^{-}$direction for the reaction $K^{-} p \rightarrow \Lambda \pi^{+} \pi^{-}$with $M_{\Lambda \pi^{-}}$in the range of $1385 \pm 45 \mathrm{MeV}$ and $\cos \theta_{K \Sigma^{*}}>0.95$. For a $\Sigma^{*}$ with $J=3 / 2$, the angular distribution is expected to be of the form $\left(1+3 \cos ^{2} \theta\right) / 2$; while for a $\Sigma^{*}$ with $J=1 / 2$, a flat constant distribution is predicted. The data [28] as shown in Fig. 2 clearly favor the case of $J=3 / 2$ if only a single $\Sigma^{*}$ resonance is assumed. However, the Fit 2 with two $\Sigma^{*}$ resonances with the narrower one of $J=3 / 2$ and the broader one with $J=1 / 2$ reproduces the data even better as shown by the solid curve in Fig. 4. The results show that the inclusion of an additional $\Sigma^{*}\left(1 / 2^{-}\right)$besides the well-established $\Sigma^{*}(1385) 3 / 2^{+}$seems improving the fit to the data of $K^{-}$beam momenta around $1.4 \mathrm{GeV}$ for both $\Lambda \pi^{-}$invariant mass spectrum and the angular distribution although the large error bars for the angular distribution data make it not very conclusive.

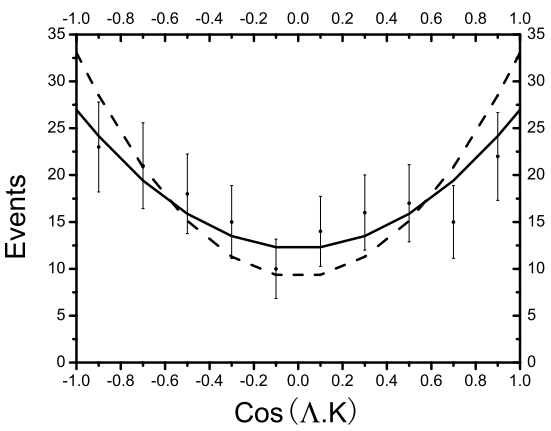

Figure 4: Predictions for the angular distribution for the reaction $K^{-} p \rightarrow \Lambda \pi^{+} \pi^{-}$at beam momenta around 1.4 $\mathrm{GeV}$ by Fit1 (dashed curve) and Fit2 (solid curve), compared with the data from Ref. [28]

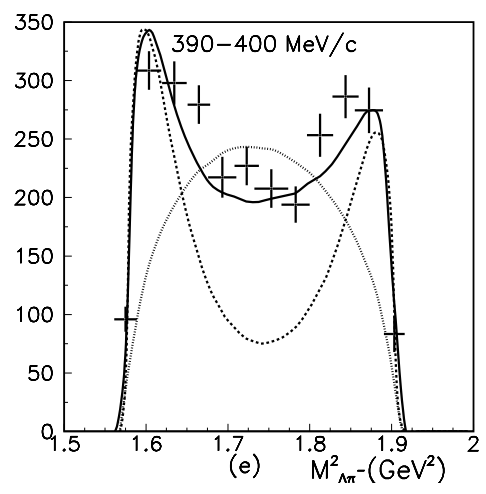

Figure 5: Theoretical $\Lambda \pi^{-}$invariant mass squared distribution with pure $\Sigma^{*}\left(3 / 2^{+}\right)$(dotted line) and with a $\Sigma^{*}\left(1 / 2^{-}\right)$ in addition (solid line) for the $K^{-} p \rightarrow \Lambda^{*}(1520) \rightarrow$ $\Sigma^{*} \pi \rightarrow \Lambda \pi^{+} \pi^{-}$reaction at beam momenta around $0.4 \mathrm{GeV}$, compared with data [30]. 
Further stronger evidence is found from a study [29] on the data of the $K^{-} p \rightarrow \Lambda^{*}(1520) \rightarrow$ $\Sigma^{*} \pi \rightarrow \Lambda \pi^{+} \pi^{-}$reaction at beam momenta around $0.4 \mathrm{GeV}$ [30]. The theoretical $\Lambda \pi^{-}$invariant mass squared distribution with pure $\Sigma^{*}\left(3 / 2^{+}\right)$fails to reproduce the data as shown by the dotted line in Fig. 5. With a $\Sigma^{*}\left(1 / 2^{-}\right)$in addition, the data are perfectly reproduced as shown by the solid line in Fig.5.

Recently, the LEPS collaboration reported a measurement of the reaction $\gamma n \rightarrow K^{+} \Sigma^{*-}(1385)$ with linearly polarized photon beam at resonance region [31]. The beam asymmetry is sizably negative at $E_{\gamma}=1.8-2.4 \mathrm{GeV}$, which is in great contrast to the theoretical prediction of a very small beam asymmetry [32]. It is found that including an additional $\Sigma^{*}\left(1 / 2^{-}\right.$gives naturally the sizably negative beam asymmetry [33].

\section{5-quark components in other baryons}

The 5-quark components in other baryons are also found important. An admixture of 10$20 \%$ of $q q q q \bar{q}$ components in the $\Delta(1232)$ resonance is shown to reduce the well known underprediction for the decay width for $\Delta(1232) \rightarrow N \gamma$ decay by about half and that of the corresponding helicity amplitudes about a factor 1.6. The main effect is due to the quark-antiquark annihilation transitions $q q q q \bar{q} \rightarrow q q q \gamma$, the consideration of which brings the ratio $A_{3 / 2} / A_{1 / 2}$ and consequently the E2/M1 ratio $R_{E M}$ into agreement with the empirical value [18]. The large contribution of the quark-antiquark annihilation transitions may also compensate the under-prediction of the $\pi N$ decay width of the $\Delta(1232)$ by the valence quark model, once the $\Delta(1232)$ contains $q q q q \bar{q}$ components with $\sim 10 \%$ probability [19]. The presence of substantial $q q q q \bar{q}$ components in the $N^{*}(1440)$ can bring about a reconciliation of the constituent quark model with the large empirical decay width of the $N^{*}(1440)$ [20, 34].

From a recent study [35] of the strong near-threshold enhancement of $p p \rightarrow n K^{+} \Sigma^{+}$cross section, the extraordinary large coupling of the $\Delta^{*}(1620)$ to $\rho N$ obtained from the $\pi^{+} p \rightarrow N \pi \pi$ is confirmed. Does the $\Delta^{*}(1620)$ contain a large $\rho N$ molecular component or relate to some $\rho N$ dynamical generated state? If so, how about its SU(3) decuplet partners? In fact, from PDG compilation [22] of baryon resonances, there are already some indications for a vector-mesonbaryon SU(3) decuplet. While the $\Delta^{*}(1620) 1 / 2^{-}$is about $85 \mathrm{MeV}$ below the $N \rho$ threshold, there is a $\Sigma^{*}(1750) 1 / 2^{-}$about $70 \mathrm{MeV}$ below the $N K^{*}$ threshold and there is a $\Xi^{*}(1950) ?^{?}$ about 60 $\mathrm{MeV}$ below the $\Lambda K^{*}$ threshold. If these resonances are indeed the members of the $1 / 2^{-} \mathrm{SU}(3)$ decuplet vector-meson-baryon $S$-wave states, we would expect also a $\Omega^{*} 1 / 2^{-}$resonance around $2160 \mathrm{MeV}$. All these baryon resonances can be searched for in high statistic data on relevant channels from vector charmonium decays by upcoming BES3 experiments in near future.

There are many other baryon resonances which are proposed to be meson-baryon states [36], i.e., $q q q q \bar{q}$ regrouping into two colorless clusters.

\section{Conclusion}

For the proton, there should be at least about $20 \%$ mixture of the five-quark components in the proton to reproduce its large $\bar{u}-\bar{d}$ asymmetry $(\bar{d}-\bar{u} \approx 0.12)$ and $s-\bar{s}$ asymmetry. To tell the relative importance of meson cloud and diquark cluster mixtures in the proton, more precise experiments on the strangeness radius and strangeness magnetic moment of the proton are needed.

There should be more five-quark components in excited baryons. The study of $1 / 2^{-}$baryons seems telling us that the $\bar{q} q q q q$ in S-state is more favorable than $q q q$ with $L=1$. In other words, 
for excited baryons, the excitation energy for a spatial excitation could be larger than to drag out a $q \bar{q}$ pair from gluon field. Whether the $\bar{q} q q q q$ components are in diquark cluster configuration or meson-baryon configuration depends on the strength of relevant diquark or meson-baryon correlations. For $N^{*}(1535)$ and its $1 / 2^{-} \mathrm{SU}(3)$ nonet partners, the diquark cluster picture for the penta-quark configuration gives a natural explanation for the longstanding mass-reverse problem of $N^{*}(1535), N^{*}(1440)$ and $\Lambda^{*}(1405)$ resonances as well as the unusual decay pattern of the $N^{*}(1535)$ resonance. For $\Delta^{*++}(1620)$ and its $1 / 2^{-} \mathrm{SU}(3)$ decuplet partners, their SU(3) quantum numbers do not allow them to be formed from two good scalar diquarks plus a $\bar{q}$. Then their $\bar{q} q q q q$ components would be mainly in meson-baryon configurations.

Distinctive patterns are predicted by quenched quark models and unquenched quark models for the lowest $\mathrm{SU}(3)$ baryon nonet with spin parity $J^{P}=1 / 2^{-}$. While the quenched quark models predict the lowest $1 / 2^{-} \Sigma^{*}$ resonance to be above $1600 \mathrm{MeV}$, the unquenched quark models predict it to be around $\Sigma^{*}(1385)$ energy. By re-examining some old data of the $K^{-} p \rightarrow \Lambda \pi^{+} \pi^{-}$ reaction, it is found that besides the well established $\Sigma^{*}(1385)$ with $J^{P}=3 / 2^{+}$, there is indeed some some evidence for the possible existence of a new $\Sigma^{*}$ resonance with $J^{P}=1 / 2^{-}$around the same mass but with broader decay width. There are also indications for such possibility in the $J / \psi \rightarrow \bar{\Sigma} \Lambda \pi$ and $\gamma n \rightarrow K^{+} \Sigma^{*-}$ reactions. At present, the evidence is not very strong. Therefore, high statistics studies on the relevant reactions, such as $K^{-} p \rightarrow \pi \Sigma^{*}, \gamma N \rightarrow K \Sigma^{*}, \psi \rightarrow \bar{\Sigma} \Sigma^{*}$ with $\Sigma^{*} \rightarrow \Lambda \pi$ or $\Sigma \pi$, are urged to be performed by forthcoming experiments at JPARC, CEBAF, BEPCII, etc., to clarify the situation.

Acknowledgements I would like to thank D.O.Riska, B.C.Liu, J.J.Xie, C.S.An, J.J.Wu, F.X.Wei, X.Cao, S.Dulat and H.C.Chiang for collaborations on relevant issues reported here, and thank the Institute for Nuclear Theory at the University of Washington for its hospitality during the completion of this paper. This work is partly supported by the National Natural Science Foundation of China (NSFC) under grants Nos. 10875133, 10821063, 10635080, and by the Chinese Academy of Sciences (KJCX3-SYW-N2), and by the Ministry of Science and Technology of China (2009CB825200).

\section{References}

[1] G.T. Garvey, J.C. Peng, Prog. Part. Nucl. Phys. 47 (2001) 203, and references therein.

[2] J.P. Speth, A.W. Thomas, Adv. Nucl. Phys. 24 (1997) 93, and references therein.

[3] B.S. Zou, D.O. Riska, Phys. Rev. Lett. 95 (2005) 072001; D.O. Riska, B.S. Zou, Phys. Lett. B 636 (2006) 265; C.S. An, D.O. Riska, B.S. Zou, Phys. Rev. C 73 (2006) 035207.

[4] F.X. Wei, B.S. Zou, Phys. Lett. B 660 (2008) 501; arXiv:0807.2324 [hep-ph].

[5] D. H. Beck and R. D. McKeown, Ann. Rev. Nucl. Part. Sci. 51 (2001) 189, and references therein.

[6] R. Young et al., Phys. Rev. Lett. 97 (2006) 102002.

[7] S. Baunack et al., Phys. Rev. Lett. 102 (2009) 151803.

[8] B.C. Liu, B.S. Zou, Phys. Rev. Lett. 96 (2006) 042002; ibid, 98 (2007) 039102.

[9] L. S. Geng, E. Oset, B. S. Zou and M. Doring, Phys. Rev. C 79 (2009) 025203.

[10] M. Dugger et al., Phys. Rev. Lett. 96 (2006) 062001.

[11] X. Cao, X.G. Lee, Phys. Rev. C 78 (2008) 035207.

[12] J.J. Xie, B.S. Zou, H.C. Chiang, Phys. Rev. C 77 (2008) 015206.

[13] X. Cao, J. J. Xie, B. S. Zou and H. S. Xu, Phys. Rev. C 80 (2009) 025203.

[14] A. Sibirtsev et al., Eur. Phys. J. A 29 (2006) 363.

[15] A. Zhang et al., High Ener. Phys. Nucl. Phys. 29 (2005) 250.

[16] C. S. An and D. O. Riska, Eur. Phys. J. A 37 (2008) 263

[17] C. S. An and B. S. Zou, Eur. Phys. J. A 39 (2009) 195

[18] Q. B. Li and D. O. Riska, Nucl. Phys. A766 (2006) 172

[19] Q. B. Li and D. O. Riska, Phys. Rev. C73 (2006) 035201 
[20] Q. B. Li and D. O. Riska, Phys. Rev. C74 (2006) 015202

[21] C. S. An, Q. B. Li, D. O. Riska and B. S. Zou, Phys. Rev. C74 (2006) 055205, Erratum-ibid.C79 (2007) 069901.

[22] Particle Data Group, C. Amsler et al., Phys. Lett. B667 (2008) 1.

[23] B.S. Zou, Nucl. Phys. A 790 (2007) 110; Int. J. Mod. Phys. A21 (2006) 835; and references therein.

[24] D. Asner et al., Int. J. Mod. A 24 (2009) 499, arXiv:0809.1869

[25] J. J. Wu, S. Dulat and B. S. Zou, Phys. Rev. D 80 (2009) 017503.

[26] C. Helminen and D. O. Riska, Nucl. Phys. A 699 (2002) 624.

[27] D. Jido, J. A. Oller, E. Oset, A. Ramos and U. G. Meissner, Nucl. Phys. A 725 (2003) 181.

[28] D. O. Huwe, Phys. Rev. 181 (1969) 1824

[29] J. J. Wu, S. Dulat and B. S. Zou, arXiv:0909.1380 [hep-ph].

[30] T. S. Mast et al., Phys. Rev. D 7 (1973) 5.

[31] K. Hicks et al. [LEPS Collaboration], Phys. Rev. Lett. 102 (2009) 012501.

[32] Y. Oh, C. M. Ko and K. Nakayama, Phys. Rev. C 77 (2008) 045204.

[33] Puze Gao, J. J. Wu and B. S. Zou, arXiv:1001.0805[ [nucl-th].

[34] B. Julia-Diaz and D. O. Riska, Nucl. Phys. A 780 (2006) 175.

[35] J.J. Xie, B.S. Zou, Phys. Lett. B 649 (2007) 405.

[36] E. Oset et al., Chin. Phys. C 33 (2009) 1132, arXiv:0906.3801 [hep-ph]. 